\title{
Evidence of a Multicopper Oxidase in Mn Oxidation by Gaeumannomyces graminis var. tritici
}

\author{
Ian A. Thompson, Don M. Huber, and Darrell G. Schulze
}

First and second authors: Department of Botany and Plant Pathology, Purdue University, 915 State Street, Lilly Hall, West Lafayette, IN 47907; and third author: Department of Agronomy, Purdue University, 915 Lilly Hall, West Lafayette, IN 47907.

Accepted for publication 31 August 2005.

\begin{abstract}
Thompson, I. A., Huber, D. M., and Schulze, D. G. 2006. Evidence of a multicopper oxidase in Mn oxidation by Gaeumannomyces graminis var. tritici. Phytopathology 96:130-136.

Manganese (Mn) oxidation by the plant-pathogenic fungus Gaeumannomyces graminis var. tritici has been correlated with virulence in take-all disease. The mechanism of Mn oxidation has not, however, been investigated adequately. Research on bacteria and other fungi indicates that Mn

oxidation is most often the result of the activity of multicopper oxidases. To determine if G. graminis var. tritici oxidizes Mn by similar means, the Mn oxidizing factor (MOF) produced by G. graminis var. tritici was characterized by cultural, spectrophotometric, and cellulose acetate electrophoresis methods. Based on our results, the MOF is an extracellular enzyme with an estimated molecular weight of 50 to $100 \mathrm{kDa}$. Electrophoresis and spectrophotometry indicate that the MOF is a multicopper oxidase with laccase activity.
\end{abstract}

Gaeumannomyces graminis (Sacc.) Arx and Olivier var. tritici Walker is a soilborne plant-pathogenic fungus that causes take-all, a root and crown rot of Triticum aestivum (wheat) and other cereal hosts. Among other factors, severity of take-all is influenced by Mn availability $(18,26)$. Factors that increase soil Mn availability and decrease the severity of take-all and other soilborne diseases caused by the Gaeumannomyces graminis group include low soil pH (25), $\mathrm{NH}_{4}{ }^{+}-\mathrm{N}$ sources (25), high $\mathrm{Mn}$ seed content (33), Mn fertilization (19,20), and the use of Mn-efficient wheat cultivars $(49,50)$. Conversely, factors associated with low $\mathrm{Mn}$ availability such as high soil $\mathrm{pH}, \mathrm{NO}_{3}{ }^{-}-\mathrm{N}$ sources, or low soil Mn content increase the severity of take-all (25).

An additional factor in this Mn availability-disease interaction is that G. graminis var. tritici is capable of oxidizing Mn from the soluble, plant-available $\mathrm{Mn}^{2+}$ oxidation state to $\mathrm{Mn}^{3+} / \mathrm{Mn}^{4+}$ metal oxides and hydroxides, which are too insoluble to provide sufficient $\mathrm{Mn}$ for plant uptake from the soil or for physiological functions in the plant (5). Since the original documentation of $\mathrm{Mn}$ oxidation by $G$. graminis var. tritici (49), Mn oxidation has been observed in host tissue during infection (41). Additionally, Mn oxidation has been correlated with pathogen virulence. For example, G. graminis var. tritici isolates from fields exhibiting a decline in take-all disease were more likely to be classified as weak or nonoxidizers under laboratory culture conditions than were isolates taken from fields with severe take-all (38), and G. graminis var. tritici isolates with low Mn oxidation capacity caused fewer and less extensive lesions than isolates characterized as strong oxidizers (37).

The ability to oxidize $\mathrm{Mn}$ is well known in bacteria and fungi $(4,43,48)$. It is thought to be performed by both nonenzymatic and enzymatic mechanisms, although increasing evidence suggests enzymatic mechanisms predominate $(10,45)$. Manganese-oxidizing bacteria have received more attention than their fungal counterparts, and appear to rely primarily on enzymes classified as multi-

Corresponding author: I. A. Thompson; E-mail address: thompson@ purdue.edu

DOI: 10.1094/PHYTO-96-0130

(C) 2006 The American Phytopathological Society copper oxidases to facilitate Mn oxidation $(3,4,44,45)$. Although Mn oxidation in fungi has not been studied as extensively, at least two enzymes produced by fungi are reported to oxidize the element: laccase $(22,40)$ and Mn peroxidase $(14,15,23,43)$. Recently, Miyata et al. (34) confirmed that Mn oxidation in an Acremonium sp. was dependent on a laccase-like multicopper oxidase. Manganese peroxidase is found primarily in litter and wood-degrading basidiomycetes (23), while the laccases are more broadly distributed among fungi (31). Little work has focused on characterizing the Mn oxidizing factor (MOF) responsible for Mn oxidation by G. graminis var. tritici. Such information would be useful for identifying mechanisms and genes associated with Mn oxidation and further define the role of $\mathrm{Mn}$ oxidation in the virulence of G. graminis var. tritici and other plant-pathogenic fungi. The goal of this study was to determine the biochemical nature and basic properties of the MOF produced by G. graminis var. tritici and to test the hypothesis that the MOF is a laccase-like enzyme.

\section{MATERIALS AND METHODS}

Isolates and culture conditions. Two G. graminis var. tritici isolates from a large international collection were selected for these experiments based on previous screens for isolates with differential ability to oxidize $\mathrm{Mn}$. Isolate 1158-1 consistently oxidizes Mn in culture. Isolate 1079-1 does not oxidize Mn under axenic culture conditions (Fig. 1), though, as discussed later, isolate 1079-1 is capable of Mn oxidation in certain conditions. Both isolates are otherwise typical of $G$. graminis var. tritici in culture and grow at approximately the same rate.

The isolates were maintained on 1/4-strength potato dextrose agar (1/4 PDA) at $23^{\circ} \mathrm{C}$. Production of the MOF was detected by amending the culture medium with $1 \mathrm{mM} \mathrm{MnSO}_{4}$ (1/4 MnPDA). The formation of brown $\mathrm{Mn}$ oxides in the medium confirmed $\mathrm{Mn}$ oxidation activity (24). In some instances, $1 \mathrm{ml}$ of a $0.04 \%$ solution of Leucoberbelin Blue (LBB) was added to the surface of the agar plate to facilitate detection of low concentrations of $\mathrm{Mn}$ oxides, such as in the molecular weight estimation experiments. LBB turns a deep blue in the presence of $\mathrm{Mn}$ in any oxidation state higher than $\mathrm{Mn}^{2+}(1)$. 
Pathogenicity and virulence tests. Isolates were tested for pathogenicity and virulence by their ability to form lesions on wheat roots. Wheat seeds (cv. Caldwell) were agitated in a stream of deionized $\mathrm{H}_{2} \mathrm{O}$ for 20 min, surface-sterilized in a $0.8 \%$ sodium hypochlorite solution for $15 \mathrm{~min}$, and rinsed in sterile deionized $\mathrm{H}_{2} \mathrm{O}$. In the culture-plate infection assay, sterilized seeds were germinated between sterile, moist, Whatman filter disks in petri plates. After the radicles had reached $1 \mathrm{~cm}$ in length, the germlings were placed on top of actively growing cultures of isolates 1158-1 and 1079-1 on 1/4 PDA. Infection was determined visually by the presence of lesions on roots after 5 days at $23^{\circ} \mathrm{C}$. These experiments were repeated several times over the course of 2 years with each treatment being replicated at least three times. Virulence also was tested by growing germinated wheat seedlings in 50-ml centrifuge tubes packed with sterile vermiculite that had been inoculated with fungal-colonized agar plugs (8). The control treatment consisted of wheat seedlings grown in sterile, noninoculated vermiculite. Seeds were germinated between moistened, sterile filter paper before planting in the vermiculite. Virulence was determined visually by the presence or absence of dark lesions on the roots. Relative virulence was recorded using the criteria described by Mathre et al. (30) in which the degree of seedling root infection is assessed and compared between isolates. Each treatment was replicated five times and the entire experiment was repeated twice.

Effect of host tissue on Mn oxidation. To determine whether host tissue influences Mn oxidation, cultures of isolates 1158-1 and 1079-1 were grown for several days on 1/4 MnPDA, after which aseptically germinated, surface-sterilized wheat seedlings were placed on top of the cultures as described in the pathogenicity and virulence assay. Mn oxidation in association with the host tissue was determined after 3 to 5 days by visually inspecting the agar for the presence of $\mathrm{Mn}$ oxides. Control treatments consisted of plates colonized by G. graminis var. tritici isolates in the absence of wheat germlings, and wheat germlings placed on agar plates in the absence of the fungal isolates. These experiments were repeated four times with treatment replication varying from two to five plates per treatment.

Extracellular nature of the MOF. To determine if Mn oxidation occurs extracellularly, isolate 1158-1 was grown on 1/4 MnPDA separated from the agar by a $0.2 \mu \mathrm{m}$ polyvinylidene difluoride (PVDF) filter membrane (Millipore, Bedford, MA) on the agar surface. Plugs from growing cultures were transferred to the membranes to prevent contact of the fungal hyphae with the agar. The growing colony and membrane were removed just before the colony margin reached the edge of the membrane. The presence of Mn oxides in the agar below was determined visually. Since these membranes effectively prevent hyphal contact with the agar, Mn oxide forming in the agar can be attributed to the action of a secreted, diffusible MOF. These experiments were repeated several times with replication varying between two and five plates.

Proteinaceous nature of the MOF. To test the proteinaceous nature of the MOF, isolate 1158-1 was grown on unamended 1/4 PDA on top of $0.2-\mu \mathrm{m}$ PVDF filter membranes. When the cultures had reached the edge of the filter membrane, the culture and membrane were removed with sterile forceps. Immediately upon removal of the membranes, agar plates were sealed with Parafilm and either (i) floated in a $70^{\circ} \mathrm{C}$ water bath for $20 \mathrm{~min}$, (ii) flooded with $10 \mathrm{ml}$ of a proteinase $\mathrm{K}$ solution $(50 \mathrm{mAU} / \mathrm{ml})$ and incubated for $30 \mathrm{~min}$ at $23^{\circ} \mathrm{C}$, or (iii) left untreated at $23^{\circ} \mathrm{C}$ as a control. After incubation, all treatments were flooded with $2 \mathrm{ml}$ of a 100 $\mu \mathrm{M} \mathrm{MnSO}_{4}$ solution and evaluated for the formation of $\mathrm{Mn}$ oxides over the course of $1 \mathrm{~h}$, a time previously shown to be adequate for the production of Mn oxides in the presence of active MOF. This experiment was repeated twice.

Molecular weight estimation. The molecular weight of the MOF was estimated by modifying the procedure used to deter- mine its extracellular nature. Instead of PVDF membranes, agar plugs were placed on top of $15,25,50$, or 100 molecular weight cut-off (MWCO) Spectra/Por cellulose ester irradiated membranes (Spectrum Medical Industries, CA). The dialysis membranes were aseptically cut into sections, sliced along their length to create flat, rectangular membrane sections, and placed on top of $1 / 4$ MnPDA plates. Cultures were allowed to grow to the edge of the membrane (4 to 6 days) before removal. The presence of $\mathrm{Mn}$ oxides in the agar was taken as evidence of the MOF having passed through a given MWCO membrane and provided an approximate molecular weight. These experiments were repeated two times with three replicates per MWCO size class.

Spectrophotometric analysis of extracellular fractions. Production of the MOF in liquid culture was lower and inconsistent compared with that in solid culture (46). Therefore, extraction directly from agar was more feasible, although more tedious and time consuming. Crude fractions containing the MOF were obtained by growing $G$. graminis var. tritici isolates on 1/4 PDA, amended with $10 \mathrm{mM}$ PIPES buffer and adjusted to $\mathrm{pH} 6.5$. Colonies were grown for 7 to 10 days or until colonies had reached the edge of the PVDF membranes, at which time the colonies and the filter membranes were removed. Fungal-cellfree agar portions from under the filter membranes were excised and frozen at $-20^{\circ} \mathrm{C}$ for 2 to $4 \mathrm{~h}$, thawed, collected in doublethickness, sterilized Miracloth (EMD Biosciences, San Diego, $\mathrm{CA}$ ), and squeezed to remove the liquid. The expelled liquid was collected in $50-\mathrm{ml}$ conical tubes and stored at $-80^{\circ} \mathrm{C}$. This crude extract was concentrated 20- to 100 -fold over a $10-\mathrm{kDa}$ MWCO membrane with a 50 or 400-ml Amicon stirred cell ultra filtration device (Millipore, Billerica, MA). Protein concentrations were measured using a Bradford Protein Assay Kit (Amersco, Solon, $\mathrm{OH})$.

Manganese oxidation was detected in crude fraction extracts on a Shimadzu dual cuvette UV-visible recording spectrophotometer (Amphotech Ltd., Beverly, MA). Absorbance at $366 \mathrm{~nm}$ was recorded every $0.2 \mathrm{~s}$ as a measure of $\mathrm{Mn}$ oxide formation (11). Absorbance was typically recorded over a period of 300 to $1,400 \mathrm{~s}$.

Reaction mixtures for the time-course assays consisted of $90 \mu \mathrm{l}$ of concentrated extract and $10 \mu \mathrm{l}$ of $1 \mathrm{mM} \mathrm{MnSO}_{4}$ added to and mixed directly in the reaction cuvette. The reference cuvette contained $90 \mu \mathrm{l}$ of crude extract and $10 \mu \mathrm{l}$ of deionized $\mathrm{H}_{2} \mathrm{O}$. To compare Mn oxidation activity from extracts collected from nonoxidizing isolate 1079-1 and oxidizing isolate 1158-1, extracts were prepared from cultures of each, and analyzed in triplicate. Controls consisted of $90 \mu \mathrm{l}$ of deionized $\mathrm{H}_{2} \mathrm{O}$ rather than culture extracts. The effect of adding increasing concentrations of $\mathrm{MnSO}_{4}$ was analyzed by adding $10 \mu \mathrm{l}$ of deionized $\mathrm{H}_{2} \mathrm{O}$ or $\mathrm{MnSO}_{4}$ for a final concentration of $0,25,50,75$, and $100 \mu \mathrm{M} \mathrm{Mn}^{2+}$ in reaction tubes containing $90 \mu \mathrm{l}$ of extract from cultures of isolate 1158-1. Finally, the effect of the copper-chelating compound, $o$-phenan-

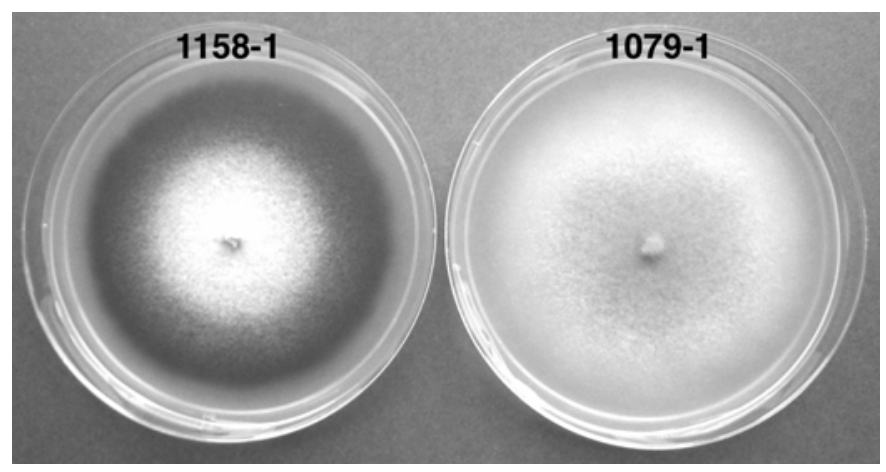

Fig. 1. Differential expression of Mn oxidation by Mn-oxidizing Gaeumannomyces graminis var. tritici isolate 1158-1 and nonoxidizing isolate 1079-1 growing on 1/4-strength potato dextrose agar supplemented with $1 \mathrm{mM} \mathrm{MnSO}_{4}$. Mn oxidation results in the deposition of brown Mn oxide precipitates. 
throline, was investigated by adding $80 \mu \mathrm{l}$ of culture extract, $10 \mu \mathrm{l}$ of $o$-phenanthroline solution $(1,10$, and $100 \mu \mathrm{M})$, and $10 \mu \mathrm{l}$ of $1 \mathrm{mM} \mathrm{MnSO}_{4}$.

Cellulose acetate electrophoresis. To test for a link between Mn oxidation and laccase activity, concentrated agar extract from cultures of $G$. graminis var. tritici isolate 1158-1 collected as described above was subjected to electrophoresis on cellulose acetate plates and stained for laccase and Mn oxidation activity. Full descriptions of cellulose acetate methods, uses, and equipment can be found in Goodwin et al. (17) and Richardson et al. (39). Briefly, samples were obtained and prepared as described for the spectrophotometric experiments. Extracts were further processed by lyophilizing $1-\mathrm{ml}$ aliquots in $1.5-\mathrm{ml}$ microcentrifuge tubes and resuspending the residue in $25 \mu \mathrm{l}$ of deionized $\mathrm{H}_{2} \mathrm{O}$. Bufferequilibrated Titan cellulose acetate plates (Helena Laboratories, Beaumont, TX) were prepared for electrophoresis by blotting $3 \mu \mathrm{l}$ of the extract onto the plates in 1- $\mu$ l aliquots to maintain small dot size. Two such applications were made on each plate with enough space between each spot to allow them to be separated by cutting the plate in half after electrophoresis so that each half could be stained with the appropriate activity indicator as described below. Further, the extract samples were blotted on the mid-line of the plate to allow migration to either the anodal or cathodal poles. Plates were marked at the edges to define the midline and allow direction of migration to be determined. Electrophoresis of the samples was conducted using a Titan Gel Chamber (Helena Laboratories) in a $5.0 \mathrm{mM}$ histidine buffer $(\mathrm{pH} 7.2)$ at 120 volts for $30 \mathrm{~min}$.

After electrophoresis, the plates were cut in half and each half was stained for either laccase or Mn oxidation activity. Laccase activity was detected using a stain overlay consisting of $2 \mathrm{ml}$ of $10 \mu \mathrm{M}$ 2,6-dimethoxyphenol (DMOP) in $2 \mathrm{ml}$ of $1.6 \%$ water agar that was poured over the plate and allowed to develop. The DMOP develops a dark orange color in the presence of laccase and has been used in other studies to detect laccase activity in G. graminis var. tritici cultures (9). Manganese oxidation activity was detected using a staining overlay consisting of $2 \mu \mathrm{l}$ of $1 \mathrm{M} \mathrm{MnSO}_{4}, 1.5 \mathrm{ml}$ of a $0.04 \% \mathrm{LBB}$ solution, and $2 \mathrm{ml}$ of $1.6 \%$ water agar. Oxidized $\mathrm{Mn}$ is detected by the formation of a dark blue color (1).

To confirm the result of these assays, a second staining method was used. In this case, one 3- $\mu$ l aliquot of culture extract was blotted onto a cellulose acetate plate and electrophoresis was conducted as described above. After electrophoresis, the plate was stained with the LBB overlay and allowed to develop for $2 \mathrm{~h}$ or until a defined blue band indicating that Mn oxides had formed.

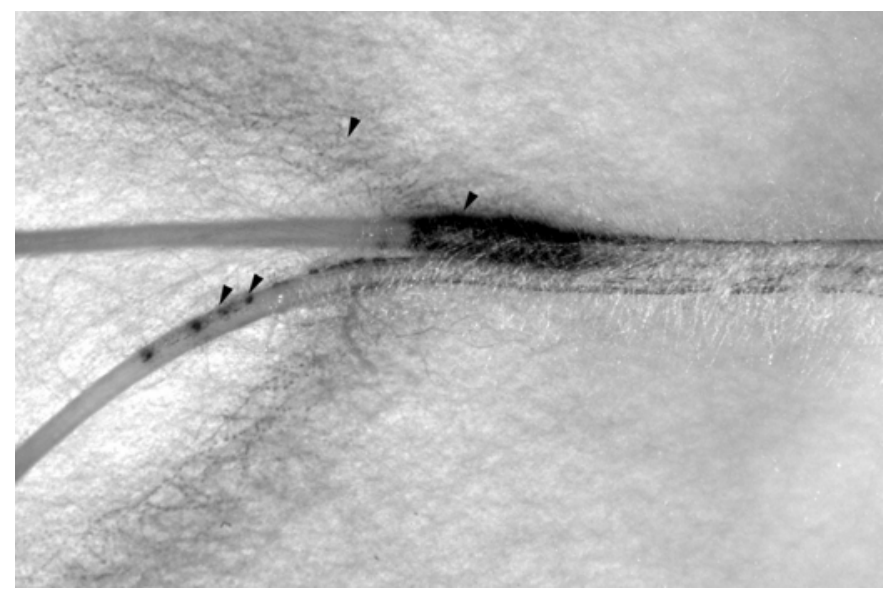

Fig. 2. Localized Mn oxidation along a wheat root: intense Mn oxidation along a wheat root infected with Gaeumannomyces graminis var. tritici isolate 1079-1. Manganese oxides accumulated directly at the point of contact between the root and the hyphal-agar interface. Oxide crusts also formed along the root surface, directly adjacent to lesions, and diffused into the surrounding agar (arrows)
At this point the LBB staining overlay was carefully removed and the same plate was treated with a DMOP staining overlay. The plate was incubated until laccase activity was clearly visible on the plate. The location of $\mathrm{Mn}$ oxidation activity in relation to laccase activity was noted.

\section{RESULTS}

Pathogenicity and virulence. Manganese oxidizing isolate 1158-1 and presumed nonoxidizing isolate 1079-1 were both pathogenic and produced lesions on wheat roots using the agarplate test. The lesions occurred primarily where roots were in contact with the agar plate, though by the end of the test they had also begun to form on portions above the surface of the agar as hyphae moved ectotopically along the root. Virulence comparisons of the isolates growing in vermiculite in conical tubes also indicated that there was no detectable difference in virulence between the isolates. Most lesions were dark brown-black, typical of those caused by highly virulent isolates (30). There was no discernable difference in virulence between these isolates using this method.

Effects of host tissue on Mn oxidation. Isolate 1158-1 did not produce noticeably different $\mathrm{Mn}$ oxidation patterns whether in the presence of plant roots or on agar without plant roots. The influence of host tissue may have been obscured due to the preexisting Mn oxides deposited in the agar by this isolate prior to placement of wheat germlings on colonized agar. Isolate 1079-1, a nonoxidizing isolate in the absence of host roots (Fig. 1), oxidized Mn immediately adjacent to the roots and on root tissue (Fig. 2). Lesions formed at the zone of contact between agar, fungus, and roots in cultures of both isolates. Control plates colonized by the fungal isolates, but lacking the host roots, gave results consistent with the isolate-specific Mn oxidization phenotypes (i.e., 1158-1 oxidized Mn and 1079-1 did not). Manganese oxides did not form in control plates lacking fungi but containing wheat germlings. These observations indicate that the Mn oxides formed on the plates inoculated with isolate 1079-1 were fungal in origin and their production was influenced by host tissue.

Extracellular nature of the MOF. The use of $0.2-\mu \mathrm{m}$ PVDF filters effectively prevented hyphae of isolate 1158-1 from contacting the agar. Extracellular components passed through the membranes and oxidized Mn. Manganese oxidation occurred in the absence of physical contact of the hyphae with the agar, which indicates that the MOF is secreted and active extracellularly.

Proteinaceous nature of the MOF. Treatment of cell-free agar medium containing the MOF with heat or proteinase $\mathrm{K}$ eliminated all $\mathrm{Mn}$ oxidation activity. Control plates retained Mn-oxidizing activity (Fig. 3). These results confirm the involvement of an enzyme in Mn oxidation by G. graminis var. tritici.

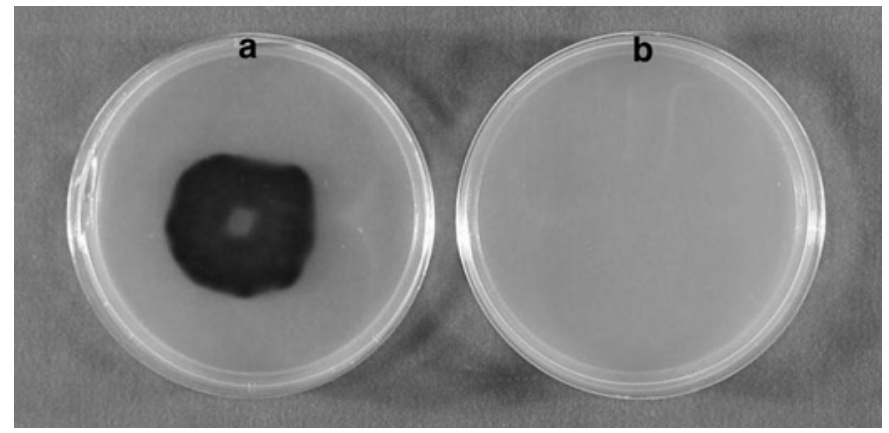

Fig. 3. Loss of Mn oxidation upon denaturation of fungal cell-free agar plate after removal of isolate 1158-1 Gaeumannomyces graminis var. tritici on a polyvinylidene difluoride membrane: a, Mn oxidation in untreated control plate; $\mathbf{b}, \mathrm{Mn}$ oxidation no longer occurs after exposure of the plates to $70^{\circ} \mathrm{C}$ for $20 \mathrm{~min}$. Exposure of plates to a proteinase $\mathrm{K}$ solution for $20 \mathrm{~min}$ also destroyed Mn oxidation ability. 
Molecular weight estimation. When isolates were grown on cellulose membranes with MWCOs of $15,25,50$, and $100 \mathrm{kDa}$, the MOF passed through the membrane and oxidized Mn in Mnamended agar only through the 50 and 100 MWCO membranes. Manganese oxidation was more intense under the $100 \mathrm{MWCO}$ membrane than the $50 \mathrm{MWCO}$ one (Fig. 4). These results suggest that the approximate molecular weight of the MOF is near $50 \mathrm{kDa}$.

Spectrophotometric analysis of extracellular fractions. Crude protein concentrations used in the analyses varied from 84 to $132 \mu \mathrm{g} / \mathrm{ml}$. The MOF was extractable from agar and the presence of the MOF could be confirmed by applying 50 to $100 \mu$ of the extract to the surface of a 1/4 MnPDA culture plate. If the MOF was present, $\mathrm{Mn}$ oxides were detected in the agar within $30 \mathrm{~min}$ of application. Manganese oxide deposits were formed by concentrated and unconcentrated extracts, but were much more obvious with concentrated extracts.

Spectrophotometric analysis of concentrated extracts from isolate 1158-1 showed increased absorbance at $366 \mathrm{~nm}$, indicating the formation of $\mathrm{Mn}$ oxides in the reaction cuvette (Fig. 5). No increase in absorbance was detected in extracts from isolate 10791 or the $\mathrm{H}_{2} \mathrm{O}$ controls. Increasing the $\mathrm{Mn}^{2+}$ concentration in the reaction mixtures increased the rate of $\mathrm{Mn}$ oxidation (Fig. 6). The addition of $o$-phenanthroline to reaction mixtures inhibited Mn oxidation (Fig. 7). This compound is a strong copper $(\mathrm{Cu})$ chelator that inactivates $\mathrm{Cu}$-containing enzymes by removing the copper prosthetic group from the enzyme or binding with copper in the enzyme and interfering with the active site. In past studies, inhibition by $o$-phenanthroline has been considered convincing

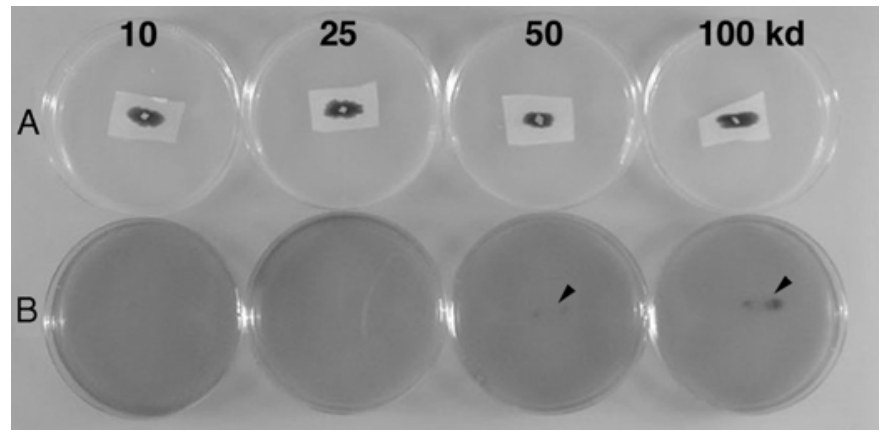

Fig. 4. Size estimation of the Mn-oxidizing factor from Gaeumannomyces graminis var. tritici. A, Isolate 1158-1 growing on 1/4-strength potato dextrose agar supplemented with $1 \mathrm{mM} \mathrm{MnSO}_{4}$ (1/4 MnPDA) over 10, 25, 50, or $100 \mathrm{kDa}$ molecular weight cut-off membranes. B, 1/4 MnPDA plate with cultures and membranes removed and flooded with Leucoberbelin Blue to enhance detection of Mn oxides in agar. Mn oxidation is detected only beneath the 50 and 100 molecular weight cut-off membranes (arrows).

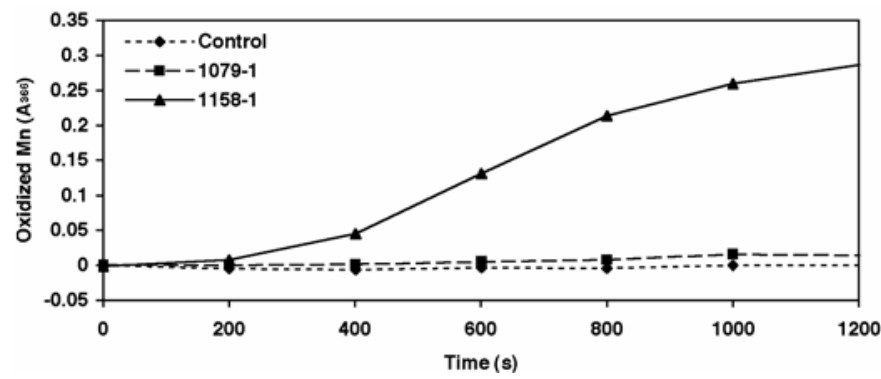

Fig. 5. Mn-oxidizing activity of oxidizing and nonoxidizing Gaeumannomyces graminis var. tritici cultures: crude fraction collected from cultures of G. graminis var. tritici isolate 1158-1 demonstrates Mn oxidation activity over $1,200 \mathrm{~s}$. Manganese oxidation activity from G. graminis var. tritici isolate 1079-1 or the deionized $\mathrm{H}_{2} \mathrm{O}$ control is negligible. Manganese oxidation is not linear in the early stages and detectable Mn oxide formation is apparent only after a lag period. Flattening of the reaction curve during the assay likely reflects a decrease in $\mathrm{Mn}^{+2}$ substrate availability. Data points are average values from three replicated time-course readings for each treatment. evidence for the involvement of a copper-containing enzyme in Mn oxidation $(4,12,13,34,36)$.

Manganese oxidation was detected after a 30- to 200-s lag period after the addition of $\mathrm{Mn}^{2+}$ (Figs. 5 to 7), with the variation in lag times probably a function of differing protein amounts in concentrated fractions. If the reaction was allowed to continue for an adequate time period, reaction rates decreased, as available $\mathrm{Mn}^{2+}$ decreased.

Cellulose acetate electrophoresis. Laccase activity co-migrated with Mn oxidation activity, both migrated to the same region of the cellulose acetate plate toward the anodal pole (Fig. 8). Laccase activity was very rapid, with visible colorimetric indication occurring as quickly as $30 \mathrm{~s}$ after application of the staining overlay. Manganese oxide detection using the LBB staining overlay developed much more slowly, sometimes requiring 2 to $3 \mathrm{~h}$ to become visible, and never became as intense as laccase activity. In the experiments where samples subjected to electrophoresis were sequentially stained for Mn oxidation followed by laccase, it was clear that $\mathrm{Mn}$ oxidation and laccase activity occupied the same zones on the plates. Laccase activity was never detected

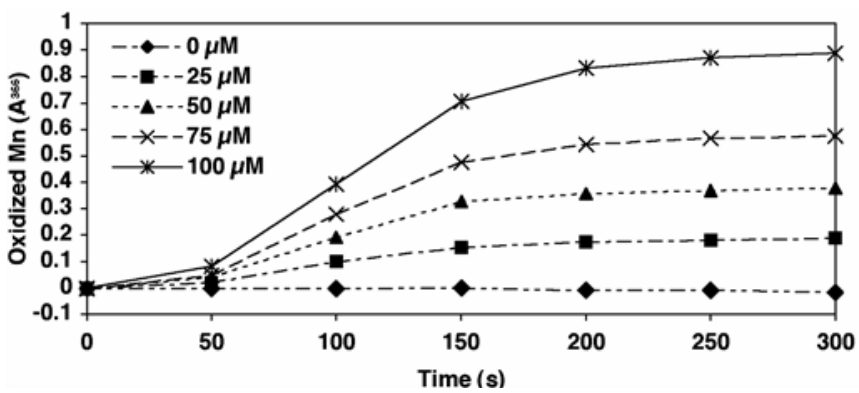

Fig. 6. Effect of Mn concentration on reaction rate. Increasing Mn-sulfate concentrations of $0,25,50,75$, and $100 \mu \mathrm{M}$ increased the rate of Mn oxidation by the Mn-oxidizing factor as measured by increasing absorbance. Data points are average values derived from three replicated time-course readings.

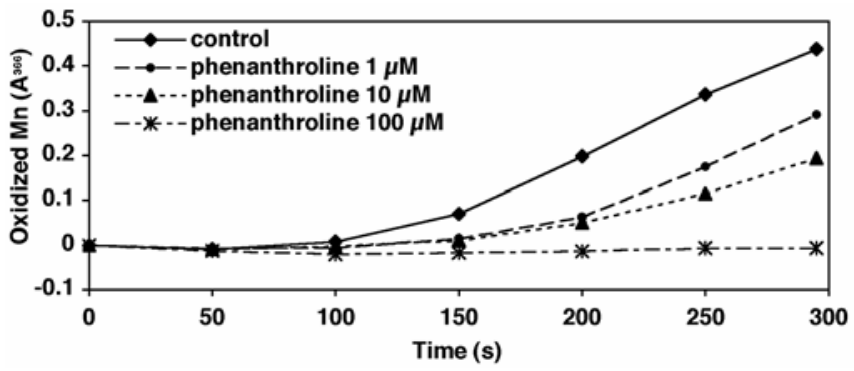

Fig. 7. Enzyme inhibition with phenanthroline. Phenanthroline, at concentrations of 1 and $10 \mu \mathrm{M}$, significantly decreased Mn oxidation, and $100 \mu \mathrm{M}$ completely eliminated oxidation compared with the uninhibited control. Data are average values derived from three replicated time-course readings for each treatment.

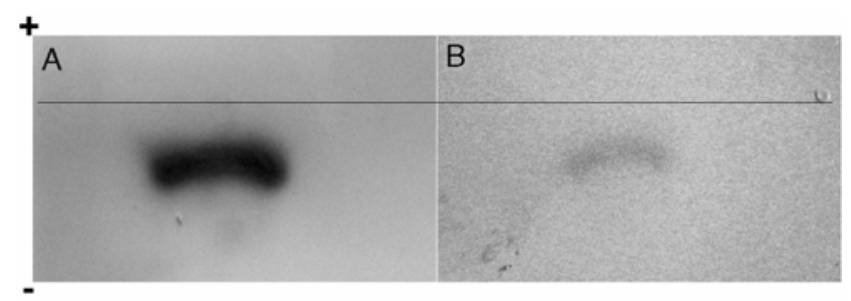

Fig. 8. Cellulose acetate plates stained for $\mathbf{A}$, laccase and $\mathbf{B}, \mathbf{M n}$ oxidation activity. The line represents the midline of the cellulose acetate gel plate at which the extracts were blotted. Laccase and Mn oxidation activity co-migrate toward the anodal pole in the electrophoretic field. Laccase activity is much more pronounced than Mn oxidation activity. 
separately from Mn-oxidation activity. This is strong evidence that an enzyme possessing laccase activity is also responsible for Mn oxidation in G. graminis var. tritici.

\section{DISCUSSION}

The results of this study indicate that the MOF is an extracellular, multicopper oxidase with laccase activity. These conclusions are based on observations that the MOF: (i) diffuses through membranes that are impenetrable to hyphae and oxidizes $\mathrm{Mn}$ in the absence of hyphae; (ii) loses activity when exposed to denaturing temperatures or the proteolytic enzyme proteinase K (Fig. 3); (iii) increases Mn oxidation rate with increasing substrate concentration $\left(\mathrm{Mn}^{2+}\right)$ (Fig. 6); (iv) is strongly inhibited by $o$-phenanthroline, a strong inhibitor of metal-containing enzymes (Fig. 7); and (v) co-migrates with laccase activity during cellulose acetate electrophoresis (Fig. 8).

The results of this study are consistent with what is currently understood about Mn oxidation by bacteria and fungi; all bacterial Mn-oxidation systems contain a multicopper oxidase system as a functional component $(4,45)$. Multicopper oxidase enzymes are associated with Mn oxidation in fungi as well, where laccase $(22,35,40)$ and a laccase-like enzyme (34) have been shown to oxidize Mn.

The strongest support for the conclusion that the MOF produced by G. graminis var. tritici is a laccase-like multicopper oxidase comes from two observations: that laccase activity co-migrates with Mn oxidation activity during cellulose acetate electrophoresis; and that Mn oxidation activity is inhibited by $o$-phenanthroline. Cellulose acetate electrophoresis is used to separate proteins based on their overall charge in a given buffer at a given $\mathrm{pH}$ (39). This method is not as harsh as sodium dodecyl sulfate-polyacrylamide gel electrophoresis and is sensitive enough to separate isozymes from crudely prepared tissues and extracts $(16,39)$. Comigration of laccase and $\mathrm{Mn}$ oxidation activity in an electrophoretic field has been demonstrated by other researchers investigating Mn oxidation by ascomycetes (34), and here, provides strong evidence that a laccase or laccase-like enzyme is responsible for Mn oxidation by $G$. graminis var. tritici.

Ortho-phenanthroline is a potent inhibitor of metallo-proteins, including multicopper oxidases and its inhibitory effect is likely due to interference with the metal centers of such enzymes (36). While $o$-phenanthroline may have decreased $\mathrm{Mn}$ oxidation by chelating $\mathrm{Mn}^{2+}$, this effect appears negligible since significant inhibition occurred with a $o$-phenanthroline concentration far below that of the $\mathrm{Mn}^{2+}$ ion concentration. This indicates that $o$-phenanthroline inhibits Mn oxidation by disrupting the copper centers of the Mn-oxidizing multicopper oxidases, as suggested by other researchers $(4,12,13,34,36)$.

Other observations in this study support the conclusion that the MOF is a multicopper oxidase, likely a laccase. For example, its extracellular nature and size estimate of 50 to $100 \mathrm{kDa}$ for the MOF is consistent with many laccases $(31,47)$, including a laccase produced by $G$. graminis var. tritici (9). Furthermore, Mn oxidation by isolate 1079-1 was upregulated by the presence of host tissue, a phenomenon also observed with some laccases produced by plant pathogens including G. graminis var. tritici $(28,29)$. Lastly, our spectrophotometric data, which demonstrates a significant lag time between the addition of $\mathrm{Mn}^{2+}$ to the reaction vessel and the initiation of Mn oxidation, is similar to observations of another ascomycete-produced laccase-like multicopper oxidase (34).

Laccases fall under the broader classification of multicopper oxidases. They have broad substrate affinities, are involved in multiple biochemical processes (31), and fungi typically harbor several laccase isozymes in their genomes $(21,27,31)$. G. graminis var. tritici is known to harbor at least three laccase genes (29). Lac1 is constitutively expressed, Lac2 appears to be upregulated in the presence of copper ions, and Lac3 is upregulated in the presence of plant host root homogenates. Our observations, and those of others $(2,25,32,37,38)$ indicate that most $G$. graminis var. tritici isolates constitutively oxidize $\mathrm{Mn}$. Therefore, our evidence supporting the involvement of a laccase in Mn oxidation makes it probable that the MOF of most G. graminis var. tritici isolates is Lac1 (9,29). While G. graminis var. tritici isolate 1079-1 does not exhibit Mn oxidation in culture, it does produce laccase activity (data not shown). Given the observation that laccase isozymes exhibit widely varied substrate specificities (31), it may be that the 1079-1 constitutively produces a laccase isozyme that does not oxidize Mn. Our observation that isolate 1079-1 oxidizes Mn in the presence of host tissue suggests that G. graminis var. tritici Lac3 also may oxidize Mn, or that another laccase, such as Lacl, is upregulated in the presence of host tissue for this isolate. In either case, the upregulation of Mn oxidation in the presence of host tissue could explain why 1079-1 is fully virulent even though initially classified as a nonoxidizer. This isolate may provide an interesting system for real-time polymerase chain reaction to further study and confirm Mn oxidation by laccase isozymes produced by specific isolates of G. graminis var. tritici (29).

Our findings supporting involvement of a laccase-like enzyme in Mn oxidation by $G$. graminis var. tritici are interesting when one considers that both laccase (31) and Mn oxidation have been separately ascribed a role in fungal virulence $(25,37,38)$. Our research suggests two mechanisms for the formation of $\mathrm{Mn}$ oxides. It may be that the formation of Mn oxides by laccase is simply a secondary phenomenon, a by-product, or an indication of some other biochemical function that influences fungal virulence and thus has been correlated with, but does not actually alter virulence. Our observation that the action of this enzyme is much stronger on the laccase substrate DMOP than on $\mathrm{Mn}^{2+}$ supports this concept. In contrast, it may be that virulence-enhancing effects of laccase are at least in part due to Mn oxidation. Oxidized forms of $\mathrm{Mn}\left(\mathrm{Mn}^{3+} / \mathrm{Mn}^{4+}\right)$ and laccases perform similar biochemical reactions that may be beneficial to pathogens invading plant hosts such as the break down or polymerization of polyphenolic compounds and detoxification of oxidative radical species $(23,31$, 44,45). It is also hypothesized that, because $\mathrm{Mn}$ is an important element in plant disease resistance (26), Mn oxidation by an invading pathogen decreases the amount of $\mathrm{Mn}$ that is available to the host for defense reactions, increasing susceptibility. Thus, it may be that the virulence-modifying reactions attributed to laccases are due in part, or are synergistically influenced by, the oxidized forms of Mn generated by some laccases.

This study helps to identify the mechanism of Mn oxidation in $G$. graminis var. tritici; however, several questions remain unanswered. For example, it is not known if the MOF oxidizes $\mathrm{Mn}^{2+}$ directly to a solid $\mathrm{Mn}$ oxide form or if an intermediate ionic $\mathrm{Mn}^{3+}$ species is formed first, or for how long the ionic $\mathrm{Mn}^{3+}$ state may persist. If $\mathrm{Mn}^{3+}$ is the initial or primary product, then $\mathrm{Mn}^{4+}$ likely forms from the auto-oxidation of $\mathrm{Mn}^{3+}$ rather than by stepwise oxidation by the enzyme itself $(44,45)$, a side reaction instead of a main product. Basidiomycete Mn-oxidizing laccase produces ionic $\mathrm{Mn}^{3+}$ in the presence of organic acids $(22,35,40)$, but investigation of the laccase-like Mn-oxidizing enzyme produced by an ascomycete indicates that $\mathrm{Mn}^{4+}$ appeared to be the primary product and no $\mathrm{Mn}^{3+}$ intermediary was detected (34).

It is also not known if only one enzyme is responsible for $\mathrm{Mn}$ oxidation. Our observation that only one band of Mn oxidation activity is visible after cellulose acetate electrophoresis suggests that there is only one active MOF product, but given that there are several laccase isozymes it may be that only one, some, or all of them are capable of $\mathrm{Mn}$ oxidation. It also is a possibility that other enzymes outside of the multicopper oxidase group are involved in Mn oxidation. If another enzyme is involved, Mn peroxidase is a likely target. The primary function of this enzyme is the formation of reactive $\mathrm{Mn}^{3+}$ ions, but it also has been 
attributed as having a role in the formation of $\mathrm{Mn}$ oxides by basidiomycetes in culture $(14,42)$. It is thought that Mn peroxidase is found exclusively in litter- and wood-degrading fungi (23). Though it was reported in an ectomycorrhizal species (7), those reports were later found to be false (6). There are no reports of this gene in other ascomycetes. Recent work in our lab, however, suggests that Magnaporthe grisea, an Mn-oxidizing, plantpathogenic ascomycete in the same family as G. graminis var. tritici, possesses an Mn peroxidase homolog that when disrupted by homologous recombination, alters, but does not entirely eliminate, Mn oxidation (46). Work on this unique gene continues. We have developed degenerative primers and are attempting to locate this $\mathrm{Mn}$ peroxidase homolog in G. graminis var. tritici and other plant-pathogenic fungi. If $G$. graminis var. tritici has a similar gene, it may be contributing to Mn oxidation as it appears to do in $M$. grisea. In either case, multiple Mn-oxidizing isozymes, or entirely different Mn-oxidizing enzymes, suggest functional redundancy which would support some biologically significant role for Mn oxidation.

Most importantly, the role of Mn oxidation in virulence in the take-all interaction remains unresolved. Although isolate 1079-1 did not oxidize $\mathrm{Mn}$ in agar culture, its ability to oxidize $\mathrm{Mn}$ in the presence of host tissue could account for it being just as virulent as isolate 1158-1 which oxidizes $\mathrm{Mn}$ in culture, and supports the hypothesis of a link between Mn oxidation and virulence (25, $37,38)$. This suggests that simple culture tests to assess the association between Mn oxidation and virulence may be flawed. Manganese oxidation by plant pathogens and its role in pathogenesis and virulence deserves further scrutiny.

\section{ACKNOWLEDGMENTS}

Journal article 17,446 of the Purdue Agriculture Research Station. This research was supported by the U.S. Department of Agriculture, National Research Initiative (grant 01-35107-11143) and by the Department of Botany and Plant Pathology at Purdue University. We thank our colleagues in the Botany and Plant Pathology Department at Purdue University for their insight, material assistance, and useful suggestions.

\section{LITERATURE CITED}

1. Adams, L. F., and Ghiorse, W. C. 1987. Characterization of extracellular $\mathrm{Mn}^{2+}$ oxidizing activity and isolation of an $\mathrm{Mn}^{2+}$ oxidizing protein from Leptothrix discophora SS-1. J. Bacteriol. 169:1279-1285.

2. Arnott, H. J., Roseman, T. S., Graham, R. D., and Huber, D. M. 1991. An experimental study of Mn oxidation mineralization in the take-all fungus Gaeumannomyces graminis var. tritici. Mycol. Soc. Am. Newsl. 42:3.

3. Bargar, J. R., Tebo, B. M., and Villinski, J. E. 2000. In situ characterization of $\mathrm{Mn}$ (II) oxidation by spores of the marine Bacillus sp. strain SG1. Geochim. Cosmochim. Acta 64:2775-2778.

4. Brouwers, G. J., Vijgenboom, E., Corstjens, P., De Vrind, J. P. M., and de Vrind-de Jong, E. W. 2000. Bacterial $\mathrm{Mn}^{2+}$ oxidizing systems and multicopper oxidases: An overview of mechanisms and functions. Geomicrobiol. J. 17:1-24.

5. Burnell, J. N. 1988. The biochemistry of manganese in plants. Pages 125133 in: Manganese in Soils and Plant. H. J. Graham, R. J. Hannam, and N. C. Uren, eds. Kluwer Academic Publishers, Boston.

6. Cairney, J. W. G., Taylor, A. F. S., and Burke, R. M. 2003. No evidence for lignin peroxidase genes in ectomycorrhizal fungi. New Phytol. 160:455-462.

7. Chen, D. M., Taylor, A. F. S., Burke, R. M., and Cairney, J. W. G. 2001. Identification of genes for lignin peroxidases and manganese peroxidases in ectomycorrhizal fungi. New Phytol. 152:151-158.

8. Dufresne, M., and Osbourn, A. E. 2001. Definition of tissue-specific and general requirements for plant infection in a phytopathogenic fungus. Mol. Plant-Microbe Interact. 14:300-307.

9. Edens, W. A., Goins, T. Q., Dooley, D., and Henson, J. M. 1999. Purification and characterization of a secreted laccase of Gaeumannomyces graminis var. tritici. Appl. Environ. Microbiol. 65:3071-3074.

10. Ehrlich, H. L. 1999. Microbes as geologic agents: Their role in mineral formation. Geomicrobiol. J. 16:135-153.

11. Emerson, D., and Ghiorse, W. C. 1992. Isolation, cultural maintenance, and taxonomy of a sheath-forming strain of Leptothrix discophora and characterization of manganese oxidizing activity associated with the sheath. Appl. Environ. Microbiol. 58:4001-4010.

12. Francis, C. A., Casciotti, K. L., and Tebo, B. M. 2002. Localization of $\mathrm{Mn}$ (II)-oxidizing activity and the putative multi-copper oxidase, MnxG, to the exosporium of the marine Bacillus sp. strain SG-1. Arch. Microbiol. 178:450-456.

13. Francis, C. A., and Tebo, B. M. 2001. CumA multi-copper oxidase genes from diverse $\mathrm{Mn}(\mathrm{II})$-oxidizing and non-Mn(II)-oxidizing Pseudomonas strains. Appl. Environ. Microbiol. 67:4272-4278.

14. Glenn, J. K., Akileswaran, L., and Gold, M. H. 1986. Mn(II) oxidation is the principal function of the extracellular Mn-peroxidase from Phanerochaete chrysosporium. Arch. Biochem. Biophys. 251:688-696.

15. Gold, M. H., and Glenn, J. K. 1988. Manganese peroxidase from Phanerochaete chrysosporium. Methods Enzymol. 161:258-264.

16. Goodwin, S. B. 2003. Isozyme analysis in fungal taxonomy, genetics, and population biology. Pages 37-47 in: Fungal Biotechnology in Agricultural, Food, and Environmental Applications. D. K. Arora, ed. Marcel Dekker, Inc., New York.

17. Goodwin, S. B., Schneider, R. E., and Fry, W. E. 1995. Cellulose acetate electrophoresis provides rapid identification of allozyme genotypes of Phytophthora infestans. Plant Dis. 79:1181-1185.

18. Graham, R. D., and Webb, M. J. 1991. Micronutrients and disease resistance and tolerance in plants. Pages 329-370 in: Micronutrients in Agriculture. J. J. Mortvedt, F. R. Cox, L. M. Shuman, and R. M. Welch, eds. Soil Sci. Soc. Am., Madison, WI.

19. Heckman, J. R., Clarke, B. B., and Murphy, J. A. 2003. Optimizing manganese fertilization for the suppression of take-all patch disease on creeping bentgrass. Crop Sci. 43:1395-1398.

20. Hill, W. J., Heckman, J. R., Clarke, B. B., and Murphy, J. A. 1999. Takeall patch suppression in creeping bentgrass with manganese and copper. HortScience 34:891-892.

21. Hoegger, P. J., Navarro-Gonzalez, M., Kilaru, S., Hoffmann, M., Westbrook, E. D., and Kues, U. 2004. The laccase gene family in Coprinopsis cinerea (Coprinus cinereus). Curr. Genet. 45:9-18.

22. Hofer, C., and Schlosser, D. 1999. Novel enzymatic oxidation of $\mathrm{Mn}^{2+}$ to $\mathrm{Mn}^{3+}$ catalyzed by a fungal laccase. FEBS Lett. 451:186-190.

23. Hofrichter, M. 2002. Review: Lignin conversion by manganese peroxidase (MnP). Enzyme Microbiol. Tech. 30:454-466.

24. Huber, D. M., and Graham, R. D. 1992. Techniques for studying nutrientdisease interactions. Pages 204-214 in: Methods for Research on Soilborne Phytopathogenic Fungi. L. L. Singleton, J. D. Mihail, and C. M. Rush, eds. The American Phytopathological Society, St. Paul, MN.

25. Huber, D. M., and McCay-Buis, T. S. 1993. A multiple component analysis of the take-all disease of cereals. Plant Dis. 77:437-447.

26. Huber, D. M., and Wilhelm, N. S. 1988. The role of manganese in resistance to plant diseases. Pages 155-173 in: Manganese in Soils and Plants. R. D. Graham, R. J. Hannam, and N. C. Uren, eds. Kluwer Academic Publishers, Dordrecht.

27. Kumar, S. V. S., Phale, P. S., Durani, S., and Wangikar, P. P. 2003. Combined sequence and structure analysis of the fungal laccase family. Biotechnol. Bioengin. 83:386-394.

28. Kwon, S. I., and Anderson, A. J. 2001. Laccase isozymes: Production by an opportunistic pathogen, a Fusarium proliferatum isolate from wheat. Physiol. Mol. Plant Pathol. 59:235-242.

29. Litvintseva, A. P., and Henson, J. M. 2002. Cloning, characterization, and transcription of three laccase genes from Gaeumannomyces graminis var. tritici, the take-all fungus. Appl. Environ. Microbiol. 68:1305-1311.

30. Mathre, D. E. 1992. Gaeumannomyces. Pages 60-63 in: Methods for Research on Soilborne Phytopathogenic Fungi. L. L. Singleton, J. D. Mihail, and C. M. Rush, eds. The American Phytopathological Society, St. Paul, MN.

31. Mayer, A. M., and Staples, R. C. 2002. Laccase: New functions for an old enzyme. Phytochemistry 60:551-565.

32. McCay, T. S. 1998. Ramifications of microbial interactions conditioning take all of wheat. Ph.D. diss. Botany and Plant Pathology Department, Purdue University, West Lafayette, IN.

33. McCay-Buis, T. S., Huber, D. M., Graham, R. D., Phillips, J. D., and Miskin, K. E. 1995. Manganese seed content and take-all of cereals. J. Plant Nutr. 18:1711-1721.

34. Miyata, N., Tani, Y., Iwahori, K., and Soma, M. 2004. Enzymatic formation of manganese oxides by an Acremonium-like hyphomycete fungus, strain KR21-2. FEMS Microbiol. Ecol. 47:101-109.

35. Munoz, C., Guillen, F., Martinez, A. T., and Martinez, M. J. 1997. Laccase isoenzymes of Pleurotus eryngii: Characterization, catalytic properties, and participation in activation of molecular oxygen and $\mathrm{Mn}^{2+}$ oxidation. Appl. Environ. Microbiol. 63:2166-2174.

36. Okazaki, M., Sugita, T., Shimizu, M., Ohode, Y., Iwamoto, K., de Vrindde Jong, E. W., de Vrind, J. P. M., and Corstjens, P. 1997. Partial purification and characterization of manganese oxidizing factors of Pseudomonas fluorescens GB-1. Appl. Environ. Microbiol. 63:4793-4799. 
37. Pedler, J. F., Webb, M. J., Buchhorn, S. C., and Graham, R. D. 1996. Manganese oxidizing ability of isolates of the take-all fungus is correlated with virulence. Biol. Fert. Soils 22:272-278.

38. Rengel, Z. 1997. Decreased capacity of the take-all fungus to oxidize manganous ions is associated with take-all decline. J. Plant Nutr. 20:455460.

39. Richardson, B. J., Baverstock, P. R., and Adams, M. 1986. Allozyme Electrophoresis: A Handbook for Animal Systematics and Population Studies. Academic Press, New York.

40. Schlosser, D., and Hofer, C. 2002. Laccase-catalyzed oxidation of $\mathrm{Mn}^{2+}$ in the presence of natural $\mathrm{Mn}^{3+}$ chelators as a novel source of extracellular $\mathrm{H}_{2} \mathrm{O}_{2}$ production and its impact on manganese peroxidase. Appl. Environ. Microbiol. 68:3514-3521.

41. Schulze, D. G., McCay-Buis, T., Sutton, S. R., and Huber, D. M. 1995. Manganese oxidation-states in Gaeumannomyces infested wheat rhizospheres probed by micro-XANES spectroscopy. Phytopathology 85:990994.

42. Steffen, K. T., Hofrichter, M., and Hatakka, A. 2000. Mineralization of C14-labelled synthetic lignin and ligninolytic enzyme activities of litterdecomposing basidiomycetous fungi. Appl. Microbiol. Biotechnol. 54:819-825.

43. Sterflinger, K. 2000. Fungi as geologic agents. Geomicrobiol. J. 17:97-124.

44. Tebo, B. M., Bargar, J. R., Clement, B. G., Dick, G. J., Murray, K. J.,
Parker, D., Verity, R., and Webb, S. M. 2004. Biogenic manganese oxides: Properties and mechanisms of formation. Annu. Rev. Earth Plant Sci. 32:287-328.

45. Tebo, B. M., Ghiorse, W. C., van Waasbergen, L. G., Siering, P. L., and Caspi, R. 1997. Bacterially mediated mineral formation: Insights into manganese(II) oxidation from molecular genetic and biochemical studies. Pages 225-266 in: Geomicrobiology: Interactions Between Microbes and Minerals. The Mineralogical Society of America, Washington, D.C.

46. Thompson, I. 2004. Manganese oxidation in plant pathogenic fungi. Ph.D. diss. Botany and Plant Pathology Department, Purdue University, West Lafayette, IN.

47. Thurston, C. F. 1994. The structure and function of fungal laccases. Microbiology 140:19-26.

48. Timonin, M. I. 1972. Oxidation of manganous salts of manganese by soil fungi. Can. J. Microbiol. 18:793-799.

49. Wilhelm, N. S. 1988. Investigations of Gaeumannomyces graminis var. tritici in Mn deficient wheat. Ph.D. diss. University of Adelaide, Adelaide, Australia.

50. Wilhelm, N. S., Graham, R. D., and Rovira, A. D. 1990. Control of Mn status and infection rate by genotype of both host and pathogen in the wheat take-all interaction. Pages 413-421 in: Genetic Aspects of Plant Mineral Nutrition. N. L. Bassam, ed. Kluwer Academic Publishers, Dordrecht, the Netherlands. 